APL

\title{
Extraction of Carrier Lifetime in Ge Waveguides using Pump Probe Spectroscopy
}

\author{
S. A. Srinivasan, ${ }^{1,2,3, \text { a) }}$ M. Pantouvaki, ${ }^{1}$ P.Verheyen, ${ }^{1}$ G. Lepage, ${ }^{1}$ P. Absil, ${ }^{1}$ J. Van Campenhout, ${ }^{1}$ and D. Van \\ Thourhout ${ }^{1,2,3, b)}$ \\ 1) Imec, Kapeldreef 75, Heverlee-3001, Belgium \\ ${ }^{2)}$ Photonics Research Group, Dept. of Information Technology, Ghent University-imec, St. Pieternieuwstraat 41, \\ Ghent-9000, Belgium \\ ${ }^{3)}$ Center for Nano- and Biophotonics, Ghent University, St. Pieternieuwstraat 41, Ghent-9000, \\ Belgium
}

(Dated: 11 February 2016)

Carrier lifetimes in Ge-on-Si waveguides are deduced using time-resolved infrared transmission pump-probe spectroscopy. Dynamics of pump-induced excess carriers generated in waveguides with varying Ge thickness and width are probed using a CW laser. The lifetimes of these excess carriers strongly depend on the thickness and width of the waveguide due to defect assisted surface recombination. Interface recombination velocities of $0.975 \times 10^{4} \mathrm{~cm} / \mathrm{s}$ and $1.45 \times 10^{4} \mathrm{~cm} / \mathrm{s}$ were extracted for the $\mathrm{Ge} / \mathrm{Si}$ and the $\mathrm{Ge} / \mathrm{SiO}_{2}$ interfaces respectively.

Optical transceivers based on silicon photonics are being considered as an alternative to their electrical counterparts for short reach interconnects. 1 Silicon photonics benefits from CMOS infrastructure to fabricate optical circuits consisting of high-speed photodetectors ${ }^{2}$, modulators 3 and filters 4 . Ge-on-Si waveguides (WG) with a p-i-n diode have shown to be a promising component in realizing high-speed and high-responsivity photodetectors $^{2}$ as well as low power consumption electro-absorption modulators ${ }^{3}$. Carrier injection based devices, such as variable optical attenuator (VOA) and Ge lasers, offer the possibility of having a complete set of Ge based active elements! ${ }^{[5}$ However, for these devices, carrier lifetimes in the WGs are important to determine the attenuation and power efficiency of the VOA as well as the threshold current density of the laser. ${ }^{67}$ Therefore, we present a method based on pump-probe transmissionspectroscopy applicable to integrated WGs to study the impact of surface recombination on carrier lifetime in Geon-Si WGs.

A set of Ge-on-Si WGs with varying width $(0.4 \mu \mathrm{m}$, $0.6 \mu \mathrm{m}, 0.8 \mu \mathrm{m}$ and $1.0 \mu \mathrm{m})$ and thickness $(0.200 \mu \mathrm{m}$ and $0.375 \mu \mathrm{m})$ is studied in this work. WGs of lengths 8 $\mu \mathrm{m}, 17.6 \mu \mathrm{m}$ and $40 \mu \mathrm{m}$ were used to model the carrier dynamics, but, only the $8 \mu \mathrm{m}$ long WGs were used to extract the carrier lifetime as will be explained later in this work. The devices were fabricated using imec's $200 \mathrm{~mm}$ Si photonics platform using a $130 \mathrm{~nm}$ CMOS toolset on $200 \mathrm{~mm}$ SOI wafer with $220 \mathrm{~nm}$ of top Si layer and $2 \mu \mathrm{m}$ buried oxide. ${ }^{8}$ For the $0.200 \mu \mathrm{m}$ and $0.375 \mu \mathrm{m}$ thick WGs, Ge was selectively grown on $30 \mathrm{~nm}$ and $110 \mathrm{~nm}$ deep recessed Si respectively. The growth was performed using reduced pressure chemical vapour deposition (RPCVD) $\underline{[}$ The wafer was annealed to suppress the threading dislocation density and, using chemical-mechanical polishing, the Ge islands were planarized to their target thickness.

\footnotetext{
a) Electronic mail: ashwyn.srinivasan@imec.be

b) Electronic mail: dries.vanthourhout@intec.ugent.be
}

The top surface was passivated with the deposition of PECVD $\mathrm{SiO}_{2}{ }^{8}$ Poly-Si tapers were used for adiabatic optical coupling from the Ge-on-Si WG to the SOI WG. Coupling to and from the chip was performed by using TE-mode fiber grating couplers. Fig. 1 shows a crosssection scanning electron micrograph (SEM) and an optical micrograph of a $0.375 \mu \mathrm{m}$ thick Ge-on-Si WG.

The pump-probe measurements were performed using a mode-locked femtosecond fiber laser (from Calmer laser) as the pump and a CW tunable laser source as the probe. The pump has a center wavelength of $1548.57 \mathrm{~nm}$ with a pulse width of $<1$ ps at the output of the fiber. In order to determine the appropriate probe wavelength, knowledge about the band-structure of Ge in the waveguide is necessary. This is estimated by using a Joint Density of States (JDOS) model to fit the absorption coefficient of Ge in the WG ${ }^{7}$ Transmission measurements were performed using a CW tunable laser source. Fig. 2 shows the extracted absorption coefficient for a $1 \mu \mathrm{m}$ wide, $0.375 \mu \mathrm{m}$ thick and $8 \mu \mathrm{m}$ long Ge-on-Si WG after

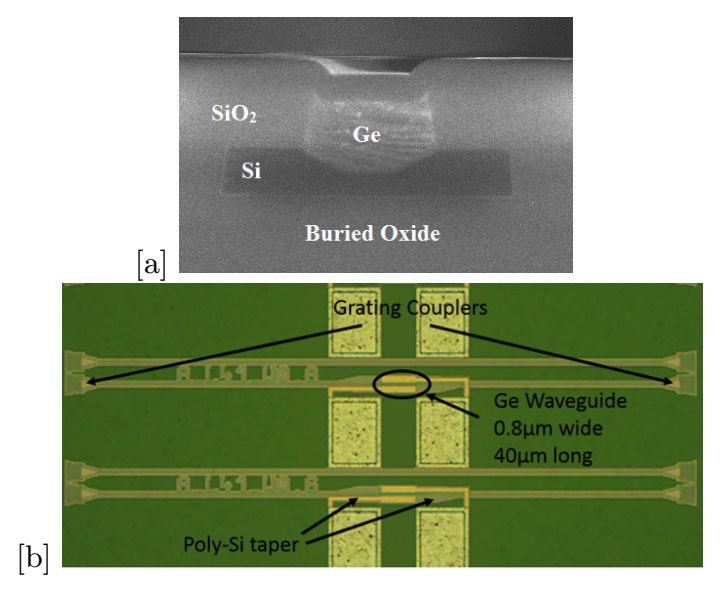

FIG. 1. a. Cross-section scanning electron micrograph of 0.8 $\mu \mathrm{m}$ wide and $0.375 \mu \mathrm{m}$ thich Ge-on-Si WG. b. Microscope image of the Device under test (DUT). 


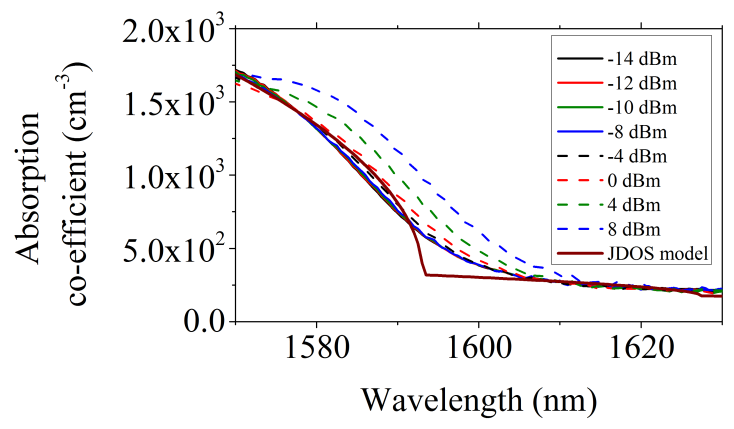

FIG. 2. Extracted absorption coefficient of Ge-on-Si WG for different laser input powers. At high laser input powers, the absorption coefficient is higher due FCA from probe-induced excess carriers. The absorption coefficient at low input power was used to fit a JDOS model to extract biaxial tensile strain and band-edge information of the Ge.

accounting for the coupling loss from a reference SOI WG and grating couplers. The coupling loss from the polySi tapers was estimated using the cut-back method. The impact of laser input power on extracting the absorptioncoefficient evidences the presence of probe-induced excess carriers that interfere with the measurement due to freecarrier absorption (FCA) and optical bleaching effects (OBE). As a result, absorption coefficients extracted at low input laser power were used to fit the JDOS model.

For bulk Ge, Jifeng Liu et. al modeled the absorption due to $\Gamma$-lh and $\Gamma$-hh transitions using the JDOS model with $E_{g, \Gamma-l h}$ and $E_{g, \Gamma-h h}$ as the band-gaps for direct conduction band $(\Gamma)$-light hole (lh) and $\Gamma$-heavy hole (hh) valley respectively. ${ }^{[7}$ These band-gaps are influenced by the degree of bi-axial tensile strain in the layer and is modeled using model-solid theory developed by $C$. Van De Walle et. a $a^{[9}$. Since TE grating couplers were used, the absorption corresponding to TE polarization will be dominant in the WG. The momentum matrix element reveals that the absorption due to $\Gamma$-hh transition is only TE polarization sensitive whereas the transition $\Gamma$-lh is predominantly TM polarization sensitive. Moreover, the sensitivity depends on the degree of bi-axial strain 10 Taking this into account, TE based absorption can be simplified to Eq. 1 where $f_{z}(\varepsilon)$ is defined by $R$. K. Schaevitz et. a ${ }^{[10}$. Indirect conduction valley mediated absorption is modelled using Macfarlane equations. ${ }^{11}$

$$
\begin{aligned}
\alpha_{T E}(h \nu, \varepsilon)= & \alpha_{E_{g, \Gamma-h h}}(h \nu, \varepsilon)+ \\
& \alpha_{E_{g, \Gamma-l h}}(h \nu, \varepsilon) \frac{1-f_{z}(\varepsilon)}{1+f_{z}(\varepsilon)}+ \\
& \alpha_{\text {indirect }}(h \nu, \varepsilon)
\end{aligned}
$$

For a $1 \mu \mathrm{m}$ wide and $0.375 \mu \mathrm{m}$ thick Ge-on-Si WG, the confinement factor for the fundamental TE mode is $73 \%$ as calculated from FemSIM ${ }^{T M}$ mode solver of RSOFT. Using Eq. 1, the confinement factor and the JDOS absorption model, a biaxial tensile strain of $0.26 \%$
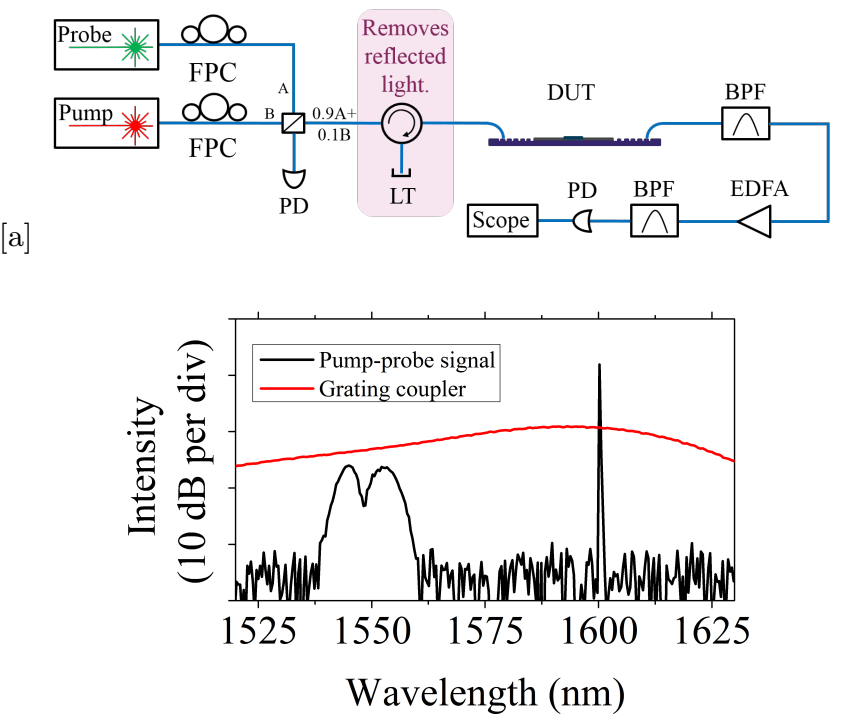

[b]

FIG. 3. a) Measurement schematic with band pass filters (BPF) centered at the probe wavelength to filter out the pump and noise from L-band EDFA with respect to the probe signal. b) The spectrum of probe signal (centered at $1600 \mathrm{~nm}$ ), pump signal (centered at $1548.57 \mathrm{~nm}$ ) and grating coupler efficiency.

was estimated in the Ge-on-Si WG. This corresponds to $E_{g, \Gamma-l h}=0.762 \mathrm{eV}(1627 \mathrm{~nm})$ and $E_{g, \Gamma-h h}=0.778 \mathrm{eV}$ $(1593 \mathrm{~nm})$. Since the absorption is dominated by the $E_{g, \Gamma-h h}$ transition for wavelengths below $1593 \mathrm{~nm}$, the probe wavelength for the experiment was set to $1600 \mathrm{~nm}$ as shown in Fig. 3 The wavelength of peak coupling for the grating coupler was set to $1595 \mathrm{~nm}$ by adjusting the fiber-chip to $13^{\circ}$ from normal incidence to allow maximum coupling for the probe signal.

The time-resolved pump-probe spectroscopy experiment is schematically shown in Fig. $33^{12}$ Here, the pump was operated at a repetition rate of $20 \mathrm{MHz}$ with an average output power of $7.9 \mathrm{~mW}$ and has an energy of 400 pJ per pulse. The probe was set to an output power of $0 \mathrm{dBm}$ at $1600 \mathrm{~nm}$ as shown in Fig. 3 . The polarization of the pump and probe beam were independently controlled for maximum coupling through the grating couplers. They were both combined using a $90 \%: 10 \%$ splitter-coupler leading to $0.5 \mathrm{~dB}$ attenuation in the probe signal and $10 \mathrm{~dB}$ attenuation in the pump signal. Such high attenuation of the pump signal reduces self-phase modulation along the optical path and is necessary to avoid two-photon absorption in the SOI WG that could generate photo-excited carriers and interfere with the measurement. Two band-pass filters (BPF) were used after the chip, to suppress the pump signal from the probe and the noise from the L-band EDFA used to amplify the probe signal. The band-pass filters were centered at the probe wavelength. An ultrafast photodiode - UPD-15-IR2-FC from ALPHALAS and Tektronix CSA8000 Signal Analyzer (Oscilloscope) were used to extract the time-resolved transmission data. The 


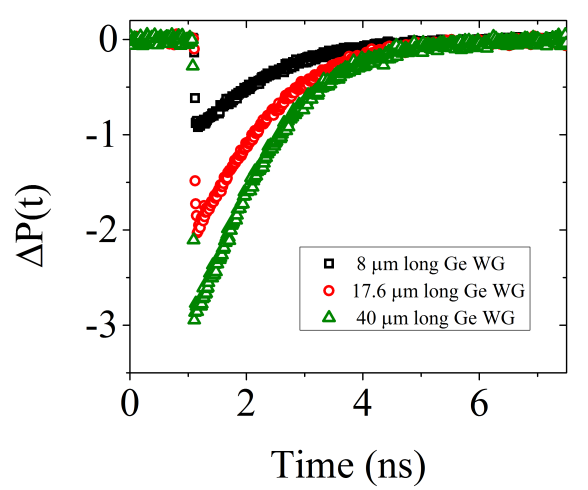

FIG. 4. Time-resolved transmission data of the probe signal from a $1 \mu \mathrm{m}$ wide $0.375 \mu \mathrm{m}$ thick Ge-on-Si WG of varying lengths. $\Delta \mathrm{P}(\mathrm{t})$ is the excess loss experienced by the probe signal through the Ge-on-Si WG due to pump beam.

oscilloscope was triggered by the pump laser. The resolution of the experiment is $<15 \mathrm{ps}$ as the bandwidth of the photo-diode and the oscilloscope are $>25 \mathrm{GHz}$.

The absorption of the pump pulses in the Ge-on-Si WG generates a high density of photo-excited electrons and holes that decay in time due to recombination. The decay of these pump-excited carriers is probed with the $1600 \mathrm{~nm} \mathrm{CW}$ signal through monitoring the excess loss from pump-induced FCA and OBE. The temporal behaviour of the probe power is monitored using the ultra fast photo-diode and the oscilloscope. Typical results extracted for a $1 \mu \mathrm{m}$ wide and $0.375 \mu \mathrm{m}$ thick Ge-on-Si WG of $8 \mu \mathrm{m}, 17.6 \mu \mathrm{m}$ and $40 \mu \mathrm{m}$ lengths are shown in Fig. 4 . In order to quantitatively extract carrier lifetime in these Ge-on-Si WG, we correlate this transmission data to the carrier density. This correlation is done by modeling the FCA and OBE losses that the probe experiences in the presence and absence of the pump beam using the JDOS model according to the equations in $2 \mathrm{a}$.

$$
\begin{aligned}
\left.\Delta P_{\text {out }}(t)\right|_{d B}= & -\left(\alpha_{F C A}(t)+\alpha_{G e}(t)-\alpha_{F C A}\left(t_{0}\right)\right. \\
& \left.-\alpha_{G e}\left(t_{0}\right)\right) \times L_{W G} \times 10 \log _{10} e
\end{aligned}
$$

where $\left.\Delta P\right|_{d B}(t)=10 \times \log _{10}\left(P_{\text {out }}(t) / P_{\text {out }}\left(t_{0}\right)\right), P_{\text {out }}\left(t_{0}\right)$ and $P_{\text {out }}(t)$ are the output power level of the probe signal at time instant $t_{0}=0$ ns and $t>0$ ns respectively. Time dependent absorption originates from FCA and intrinsic material absorption. The FCA that the $1600 \mathrm{~nm}$ probe signal endures due to pump/probeinduced free carriers $N$ and $P$ is expressed as $\alpha_{F C A}(t)=$

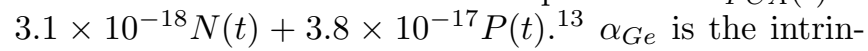
sic material absorption that takes into account optical bleaching effects due to pump/probe-induced carriers. It is mathematically expressed as

$$
\begin{aligned}
\alpha_{G e}(t) & =\alpha_{T E} \times\left(f_{v}(N(t))-f_{c}(N(t))\right) \\
& =\alpha_{T E} \times(1-O B E(N(t)))
\end{aligned}
$$

The modeling in Eq. 2b. includes the optical bleaching effect as $O B E=1-\left(f_{v}(N)-f_{c}(N)\right)$, such that if we

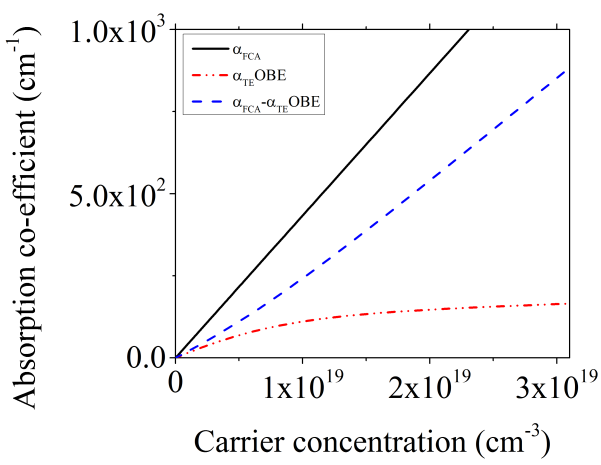

FIG. 5. Impact of photo-excited carrier on free-carrier absorption and optical bleaching effect for $1600 \mathrm{~nm}$ probe signal. $\alpha_{\text {excarr }}=\alpha_{F C A}-\alpha_{T E} O B E$ is almost linear with carrier concentration.

have no photo-excited carriers, $O B E=0$ with $f_{c}=0$ and $f_{v}=1 !^{7}$ This simplifies the Eq. $2 \mathrm{a}$ to

$$
\begin{aligned}
\left.\Delta P_{\text {out }}(t)\right|_{d B}= & -\left(\alpha_{F C A}(N(t))-\alpha_{T E} O B E(N(t))\right. \\
& \left.-\alpha_{F C A}\left(N\left(t_{0}\right)\right)+\alpha_{T E} O B E\left(N\left(t_{0}\right)\right)\right) \\
& \times L_{W G} \times 10 \log _{10} e
\end{aligned}
$$

We express the increase in absorption coefficient for the WG due to photo-excited carriers as $\alpha_{\text {excarr }}=\alpha_{F C A}-$ $\alpha_{T E} O B E$. The quasi-Fermi energy levels to estimate OBE were evaluated using a self-consistent algorithm for each assumed carrier concentration. The result of these calculations shows that $\alpha_{\text {excarr }}$ has a predominantly linear dependence with a slope $K$ as seen in Fig. 5 . We find that the time constant derived by fitting an exponential curve to $\Delta P_{\text {out }}(t)$ is the lifetime of excess carriers in the WG.

$$
\begin{aligned}
\Rightarrow \Delta P_{\text {out }}(t)= & \left(\alpha_{\text {excarr }}(N(t))-\alpha_{\text {excarr }}\left(N\left(t_{0}\right)\right)\right) \\
& \times L_{W G} \times 10 \log _{10} e \\
\Delta P_{\text {out }}(t) & \propto \Delta N(t) \\
\Delta P_{\text {max }} \exp (-t / \tau) & \propto \Delta N_{\text {max }} \exp (-t / \tau)
\end{aligned}
$$

Note that in all of the above calculations, a uniform carrier concentration is assumed across the length of the waveguide. This assumption is valid at least for the $8 \mu \mathrm{m}$ long Ge-on-Si WG as seen in Fig. 4, where $\Delta P_{\text {out }}$ for a $17.6 \mu \mathrm{m}$ long Ge-on-Si WG is approximately 2.25 times larger than the one of $8 \mu \mathrm{m}$ Ge-on-Si WG. As the pump signal is located close to the $\Gamma$-hh band-gap and has a high pump-energy, it undergoes OBE and FCA and the absorption profile deviates from the standard exponential decay. Therefore, all pump-probe experiments analysis presented in this work were performed on $8 \mu \mathrm{m}$ long Geon-Si WGs.

Fig. 6 shows the extracted $\Delta P_{\text {out }}(t)$ data after normalization with their respective $\Delta P_{\max }$. The extracted lifetimes from these curves are summarized in Fig. 7 . However, it is important to understand the dominant 

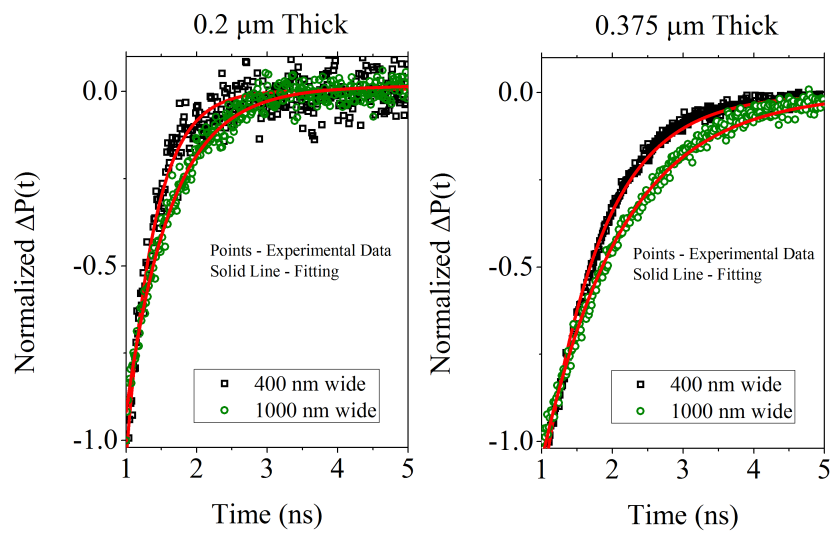

FIG. 6. Normalized $\Delta P_{\text {out }}$ decay as a function of time for 0.4 and $1.0 \mu \mathrm{m}$ wide and $0.2-0.375 \mu \mathrm{m}$ thick Ge-on-Si WGs. Each $\Delta P_{\text {out }}$ was normalized with their corresponding $\Delta \mathrm{P}_{\max }$ for extraction of carrier lifetime.

recombination phenomena limiting these lifetimes. For $\Delta \mathrm{P}_{\max }=1 \mathrm{~dB}$ in Fig. 4, the peak carrier concentration in the waveguide is approximately $2 \times 10^{19} \mathrm{~cm}^{-3}$. Assuming a radiative recombination coefficient $B=1 \times 10^{-10}$ $\mathrm{cm}^{3} \mathrm{~s}^{-1}$ and an Auger recombination coefficient $C=$ $1 \times 10^{-31} \mathrm{~cm}^{6} \mathrm{~s}^{-1,7 / 13}$ the radiative and Auger recombination limited lifetimes in the bulk are greater than $10 \mathrm{~ns}$. Therefore, the extracted lifetimes of $<1.2 \mathrm{~ns}$, as shown in Fig. 6, suggest that they are defect-assisted recombination lifetimes and not radiative or Auger recombination limited. Due to high volume/surface area ratio, we assume that bulk defects assisted recombination effects are negligible. The extracted carrier lifetimes have a strong dependence on the side walls, top and bottom interfaces of the WG. This dependence can be quantitatively expressed by evaluating the surface recombination velocity (SRV) for $\mathrm{Ge} / \mathrm{Si}$ and $\mathrm{Ge} / \mathrm{SiO}_{2} \cdot{ }^{6}$ In this study, the SRVs for the top and side $\mathrm{Ge} / \mathrm{SiO}_{2}$ interfaces were assumed to be similar for simplicity. On fitting the lifetime for each width and thickness, we extract a SRV of $0.975 \times 10^{4} \mathrm{~cm} / \mathrm{s}$ and $1.45 \times 10^{4} \mathrm{~cm} / \mathrm{s}$ for $\mathrm{Ge} / \mathrm{Si}$ and $\mathrm{Ge} / \mathrm{SiO}_{2}$ respectively. The result for the Ge/Si interface is consistent with those reproduced in the literature ${ }^{12|14| 15}$ and is attributed to the lattice mismatch between the $\mathrm{Ge}$ and the $\mathrm{Si}$. However, the SRV for the $\mathrm{Ge} / \mathrm{SiO}_{2}$ interface is found to be higher by a factor of 2-4 compared to those reported by S. A. Srinivasan et. a $i^{12}$. This may result from difference in process condition. It can be suppressed by exploring better passivation techniques for future devices. As a result, these experiments can be used to benchmark the performance of devices and aid in developing intuition for future designs. Waveguide coupled pump-probe spectroscopy techniques as presented in this paper allows the assessment of carrier lifetime in devices instead of blanket samples!1415 This technique can be used to determine the transit time of carriers in the waveguide, especially for devices such as photodetectors where the speed of the device is transit time limited and not $\mathrm{RC}$

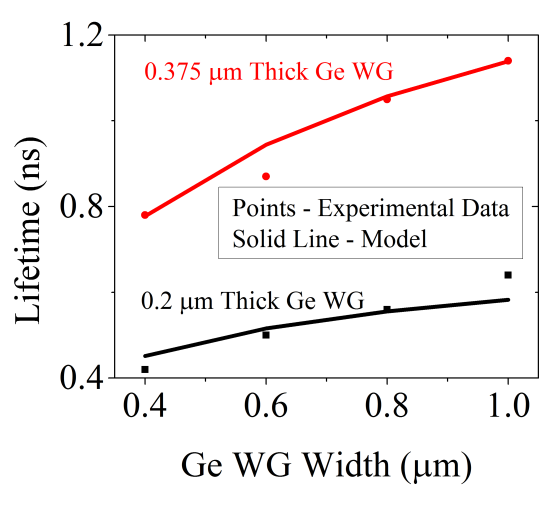

FIG. 7. Dependence of carrier lifetimes on width of the waveguide for 2 thickness extracted from Fig. 6]are shown in points. Using recombination model described by $S$. Park et. at a surface recombination velocity of $0.975 \times 10^{4} \mathrm{~cm} / \mathrm{s}$ and $1.45 \times 10^{4} \mathrm{~cm} / \mathrm{s}$ were deduced for $\mathrm{Ge} / \mathrm{Si}$ and $\mathrm{Ge} / \mathrm{SiO}_{2}$ respectively. Solid lines show the fitting of the model with the extracted lifetimes.

\section{limited $[2$}

In summary, we present a time-resolved infrared transmission pump-probe spectroscopy of Ge-on-Si waveguides. Pump-induced excess carriers generated in the waveguides are probed using a $\mathrm{CW}$ laser beam. For a 1 $\mu \mathrm{m}$ wide and $0.375 \mu \mathrm{m}$ thick Ge-on-Si WG, we extract a lifetime of $1.14 \mathrm{~ns}$. These lifetimes are dependent on the width and thickness of Ge layers. Surface recombination velocities of $0.975 \times 10^{4} \mathrm{~cm} / \mathrm{s}$ and $1.45 \times 10^{4} \mathrm{~cm} / \mathrm{s}$ are extracted for $\mathrm{Ge} / \mathrm{Si}$ and $\mathrm{Ge} / \mathrm{SiO}_{2}$ respectively. Recombination at the $\mathrm{Ge} / \mathrm{Si}$ interface is caused by lattice mismatch associated defects. With improved passivation strategies, recombination at the top and side interfaces can be suppressed to increase the lifetime of carriers in the waveguides.

This work was supported by imec's industry-affiliation program on Optical I/O. The authors would like to thank Bart Kuyken, Raphael Van Laer, Utsav Dave and Koen Alexander of Photonics Research Group for valuable discussions about the experiment.

\footnotetext{
${ }^{1}$ Miller, D.A.B., "Device Requirements for Optical Interconnects to Silicon Chips," in Proceedings of the IEEE, vol.97, no.7, pp.1166-1185, July 2009

${ }^{2}$ Hong Tao Chen; Verheyen, P.; De Heyn, P.; Lepage, G.; De Coster, J.; Absil, P.; Roelkens, G.; Van Campenhout, J., "HighResponsivity Low-Voltage 28-Gb/s Ge p-i-n Photodetector With Silicon Contacts," in Lightwave Technology, Journal of , vol.33, no.4, pp.820-824, Feb.15, 152015

${ }^{3}$ Srinivasan, S.; Pantouvaki, M.; Gupta, S.; Chen, H.; Verheyen, P.; Lepage, G.; Roelkens, G.; Saraswat, K.; Van Thourhout, D.; Absil, P.; Van Campenhout, J., "56Gb/s Germanium Waveguide Electro-Absorption Modulator," in Lightwave Technology, Journal of , vol.PP, no.99, pp.1-1

${ }^{4}$ De Heyn, P.; De Coster, J.; Verheyen, P.; Lepage, G.; Pantouvaki, M.; Absil, P.; Bogaerts, W.; Van Campenhout, J.; Van Thourhout, D., "Fabrication-Tolerant Four-Channel Wavelength-Division-Multiplexing Filter Based on Collectively Tuned Si Microrings," in Lightwave Technology, Journal of , vol.31, no.16, pp.2785-2792, Aug.15, 2013
} 
${ }^{5}$ Rodolfo E. Camacho-Aguilera, Yan Cai, Neil Patel, Jonathan T. Bessette, Marco Romagnoli, Lionel C. Kimerling, and Jurgen Michel, "An electrically pumped germanium laser," Opt. Express 20, 11316-11320 (2012)

${ }^{6}$ Sungbong Park, Koji Yamada, Tai Tsuchizawa, Toshifumi Watanabe, Hiroyuki Shinojima, Hidetaka Nishi, Rai Kou, and Sei-ichi Itabashi, "Influence of carrier lifetime on performance of silicon $\mathrm{p}-\mathrm{i}-\mathrm{n}$ variable optical attenuators fabricated on submicrometer rib waveguides," Opt. Express 18, 11282-11291 (2010)

${ }^{7}$ Jifeng Liu, Xiaochen Sun, Dong Pan, Xiaoxin Wang, Lionel C. Kimerling, Thomas L. Koch, and Jurgen Michel, "Tensilestrained, n-type Ge as a gain medium for monolithic laser integration on Si," Opt. Express 15, 11272-11277 (2007)

${ }^{8}$ Philippe P. Absil ; Peter De Heyn ; Hongtao Chen ; Peter Verheyen ; Guy Lepage ; Marianna Pantouvaki ; Jeroen De Coster ; Amit Khanna ; Youssef Drissi ; Dries Van Thourhout ; Joris Van Campenhout; "Imec iSiPP25G silicon photonics: a robust CMOS-based photonics technology platform", Proc. SPIE 9367, Silicon Photonics X, 93670V (February 27, 2015)

${ }^{9}$ Van de Walle, C. G., "Band lineups and deformation potentials in the model-solid theory", Physical Review B 39(3), 18711883, 1989.
${ }^{10}$ Cicek Boztug, Jos R. Snchez-Prez, Francesca Cavallo, Max G. Lagally, and Roberto Paiella, "Strained-Germanium Nanostructures for Infrared Photonics", ACS Nano 8 (4), 3136-3151, 2014.

${ }^{11}$ Schaevitz, R. K. and Ly-Gagnon, D. S. and Roth, J. E. and Edwards, E. H. and Miller, D. A. B.," Indirect absorption in germanium quantum wells", AIP Advances 1, 032164 (2011)

${ }^{12}$ Srinivasan, S.A.; Pantouvaki, M.; Verheyen, P.; Lepaee, G.; Absil, P.; Van Campenhout, J.; Van ThouAout, D., "Carrier lifetime assessment in integrated Ge waveguide devices," in Group IV Photonics (GFP), 2015 IEEE 12th International Conference on vol., no., pp.167-168, 26-28 Aug. 2015

${ }^{13}$ Carroll, L., Friedli, P., Neuenschwander, S., Sigg, H., Cecchi, S., Isa, F., Faist, J. (2012)," Direct-Gap Gain and Optical Absorption in Germanium Correlated to the Density of Photoexcited Carriers, Doping, and Strain." Physical Review Letters, 109(5), 057402 .

${ }^{14}$ Geiger, R., Frigerio, J., Sess, M. J., Chrastina, D., Isella, G., Spolenak, R., Sigg, H. (2014). Excess carrier lifetimes in Ge layers on Si. Applied Physics Letters 104(6), 062106.

${ }^{15}$ Donguk Nam, Ju-Hyung Kang, Mark L. Brongersma, and Krishna C. Saraswat, "Observation of improved minority carrier lifetimes in high-quality Ge-on-insulator using time-resolved photoluminescence," Opt. Lett. 39, 6205-6208 (2014) 